\title{
Increasing ankle push-off work with a powered prosthesis does not necessarily reduce metabolic rate for transtibial amputees
}

Roberto E. Quesada ${ }^{1}$

Joshua M. Caputo ${ }^{1,2}$

Steven H. Collins ${ }^{1,3}$

1. Department of Mechanical Engineering, Carnegie Mellon University, Pittsburgh, PA

2. Human Motion Technologies L.L.C., Pittsburgh, PA

3. Robotics Institute, Carnegie Mellon University, Pittsburgh, PA

Correspondence:

Steve Collins

316 Scaife Hall

Carnegie Mellon University

5000 Forbes Ave.

Pittsburgh, PA 15213

phone: 412-268-5222

email: stevecollins@cmu.edu

Keywords:

Gait, Amputation, Energetics, Robotics

Word counts:

Abstract: 241 words

Introduction through discussion: 4,044 words

Figures and tables: 1 Table, 9 Figures 


\begin{abstract}
Amputees using passive ankle-foot prostheses tend to expend more metabolic energy during walking than non-amputees, and reducing this cost has been a central motivation for the development of active ankle-foot prostheses. Increased push-off work at the end of stance has been proposed as a way to reduce metabolic energy use, but the effects of push-off work have not been tested in isolation. In this experiment, participants with unilateral transtibial amputation $(\mathrm{N}=6)$ walked on a treadmill at a constant speed while wearing a powered prosthesis emulator. The prosthesis delivered different levels of ankle push-off work across conditions, ranging from the value for passive prostheses to double the value for non-amputee walking, while all other prosthesis mechanics were held constant. Participants completed six acclimation sessions prior to a data collection in which metabolic rate, kinematics, kinetics, muscle activity and user satisfaction were recorded. Metabolic rate was not affected by net prosthesis work rate $(p=0.5$; $\left.\mathrm{R}^{2}=0.007\right)$. Metabolic rate, gait mechanics and muscle activity varied widely across participants, but no participant had lower metabolic rate with higher levels of push-off work. User satisfaction was affected by push-off work $(p=0.002)$, with participants preferring values of ankle push-off slightly higher than in non-amputee walking, possibly indicating other benefits. Restoring or augmenting ankle push-off work is not sufficient to improve energy economy for lower-limb amputees. Additional necessary conditions might include alternate timing or control, individualized tuning, or particular subject characteristics.
\end{abstract}




\section{Introduction}

Lower-limb amputation affects more than one million people in the United States (ZieglerGraham et al. 2008), leading to restricted mobility (Zidarov et al. 2009). Lower-limb amputation is typically accompanied by an increase in metabolic energy expenditure during walking (Waters and Mulroy, 1999). Reducing the energy cost of amputee gait may therefore be beneficial.

Differences between the mechanical function of biological ankles and passive-elastic prostheses could be responsible for observed increases in metabolic rate. The intact ankle joint produces a large burst of mechanical work during terminal stance (Winter, 1991), also known as 'push-off', which is diminished in passive-elastic ankle prostheses (e.g. Gitter et al., 1991). This leads to several arguments that increasing prosthetic ankle push-off work could reduce metabolic rate.

Increasing prosthetic ankle push-off work might provide a benefit by more closely matching biological ankle function. Imitating some aspect of non-amputee gait is a frequent goal of active prostheses (Au et al., 2007; Goldfarb et al., 2013). Perhaps if prostheses behaved more like biological joints, they would better fulfill their role in walking, leading to reduced effort. This leads to the hypothesis that metabolic rate should be minimized when prosthesis push-off matches the value for non-amputee gait, for example following a quadratic relationship.

Increasing prosthetic ankle push-off work might be beneficial by supplying a greater portion of the energy used in walking. If the mechanical energy requirements of walking were fixed, performing more work with the prosthesis would leave less work to be performed by the human, which might lead to less metabolic energy consumption. This principle has been proposed as a guideline for augmenting gait (the 'Augmentation Factor', Mooney et al., 2014), leading to the hypothesis that metabolic rate will decrease linearly as net prosthesis work increases.

Increasing prosthesis push-off work might also provide a benefit by reducing the mechanical work requirements of walking. Simple dynamical models of gait suggest that trailing-limb push-off work during double support reduces dissipation in 'collision' of the leading limb, thereby reducing positive center-of-mass work overall (Kuo et al., 2005). Reduced push-off would be expected to increase collision, requiring more positive work elsewhere in the gait cycle, such as through hip work during single support. This has been suggested as a reason for increased energy expenditure with passive prostheses (Houdijk et al., 2009). Increasing prosthesis push-off would decrease collision losses until they were eliminated, resulting in diminishing returns for additional prosthesis work. This leads to the hypothesis that metabolic rate will decrease exponentially as prosthesis push-off work increases, with corresponding decreases in collision work and center-of-mass work.

Studies comparing powered and passive ankle-foot prostheses suggest that increased push-off can reduce the metabolic cost of walking for amputees, but have not isolated this effect. Herr and Grabowski (2012) found that an active ankle-foot prosthesis reduced metabolic rate compared to passive-elastic prostheses, consistent with each of the above hypotheses. The device restored ankle push-off with net work from a motor, differentiating it from devices that increase push-off 
through elastic energy storage and return (Segal et al. 2012). However, many other features differed between the active and passive prostheses tested, including mass, stiffness, and dynamical properties, which might also affect metabolic rate. A more controlled experiment could discern whether net prosthesis work was responsible for the observed reduction in metabolic rate.

Controlled studies in simulation and among non-amputees suggest that metabolic rate decreases exponentially with increasing ankle push-off work. Handford and Srinivasan (2016) optimized coordination patterns in a musculoskeletal model of amputee gait, and found that metabolic rate decreased exponentially as prosthesis work increased. However, accurate model prediction of human response to new mechanical conditions is challenging (Fregly et al. 2012). Caputo and Collins (2014b) varied push-off work with a prosthesis emulator, worn by non-amputees using a simulator boot, and found that metabolic rate decreased exponentially with net prosthesis work. However, differences between amputees and non-amputees can confound comparisons of the same hardware between populations (Zelik et al., 2011). A controlled experiment performed among amputees would provide clinical relevance.

The goal of this study was to determine the relationship between prosthetic ankle push-off work and metabolic energy expended by unilateral transtibial amputees during walking. We varied push-off work over a wide range using an ankle-foot prosthesis emulator, without changing any other prosthesis features, and measured metabolic rate. Based on prevailing approaches to active prosthesis design, we hypothesized that metabolic rate would have one of three relationships with net prosthesis work rate: quadratic, with a minimum near the value for normal walking; decreasing linearly; or decreasing exponentially. A secondary goal was to investigate the underlying mechanisms affecting energy cost. We therefore measured center-of-mass mechanics, joint mechanics and muscle activity and tested for trends predicted by prior studies and models. We expected these results to provide empirical data to guide the design of active prostheses.

\section{Methods}

Six participants with unilateral transtibial amputation walked on a treadmill using a powered prosthesis emulator. Participants completed seven conditions characterized by different levels of ankle push-off work, which they experienced in six acclimation and training sessions before the final data collection. Metabolic rate, kinematics, kinetics, muscle activity and user satisfaction data were analyzed for an effect of net prosthesis work.

\subsection{Participants}

Six individuals with unilateral, transtibial amputation participated $(\mathrm{N}=6$; male; age $=47 \pm 6$ yrs; weight $=87.8 \pm 8.5 \mathrm{~kg}$; height $=1.79 \pm 0.04 \mathrm{~m}$; mean \pm s.d.; $\mathrm{K} 3$ as determined by prosthetist; Table 1). Participants were amputees for at least one year and had the ability to walk unaided at varying speeds. All participants provided written informed consent prior to participation. The protocol was approved by the Carnegie Mellon University Institutional Review Board. 


\subsection{Prosthesis emulator hardware and control}

In push-off work conditions, participants used a powered ankle-foot prosthesis emulator (Caputo and Collins 2014a) in place of their prescribed prosthetic foot (Fig. 1). The emulator had a torquecontrolled forefoot and a passive spring heel (Fig. S1; Supplementary Materials). Forefoot torque about the ankle joint was controlled by off-board motor and control hardware (Zhang et al., 2015). The emulator is capable of high peak torque and power, allowing a wide range of push-off work values to be applied without changing any other aspects of hardware between conditions.

Desired levels of net prosthesis work were achieved by controlling the relationship between ankle angle and ankle torque (Caputo \& Collins, 2014b). During dorsiflexion, the ankle acted as a stiffening spring. During plantarflexion, the ankle acted like a spring with a different torqueangle curve. This resulted in torque-angle work loops similar to those of other active prostheses (Herr \& Grabowski, 2012; Shultz et al. 2013). We chose the shape of the plantarflexion curve to achieve desired levels of net prosthesis work for each condition.

The timing of the onset of push-off corresponded to the transition between ankle dorsiflexion and ankle plantarflexion, dictated by subject movements. Dorsiflexion behavior was held constant across conditions, which tends to result in consistent push-off timing (Caputo \& Collins, 2014b). Push-off was expected to occur primarily during double support, the optimal timing observed in an experiment with non-amputees (Malcolm et al., 2015).

\subsection{Experimental protocol}

\subsubsection{Experimental conditions}

Participants completed two baseline conditions and seven push-off work conditions. In the Quiet Standing condition, participants stood neutrally. In the Prescribed condition, participants walked using their prescribed prosthesis. In powered push-off conditions, which we refer to as having Negative, Low, Medium-Low, Medium, Medium-High, High, and Highest push-off work, participants walked while the prosthesis emulator applied different levels of net prosthesis work on each step. Net prosthesis work was defined as the integral of instantaneous ankle power, such that negative work during dorsiflexion reduced net work while positive work during push-off increased it. Net prosthesis work rate was defined as net prosthesis work divided by stride period.

In powered push-off conditions, positive push-off work ranged from about half to double the value for non-amputee walking, corresponding to a net prosthesis work rate of about -2 to 10 times the value for non-amputee walking. This encompassed values for dynamic-elastic response prostheses, at the lower end of the range, and commercially-available active prostheses, in the upper half of the range. A wide range of push-off work values were applied so as to distinguish between quadratic, linear and exponential trends in metabolic rate at the high end of the spectrum. Many push-off values were tested to avoid overfitting. 


\subsubsection{Acclimation period}

Upon enrollment, participants underwent a three-day acclimation period with the prosthesis emulator. On the first day, a certified prosthetist fit and aligned the emulator according to standard procedures. During alignment, the emulator was programmed to behave like a dynamicelastic prosthesis. Treadmill speed was gradually increased to $1.25 \mathrm{~m} \cdot \mathrm{s}^{-1}$ over several walking bouts. One participant (Subject 2) could not sustain walking at this speed and instead walked at $1.1 \mathrm{~m} \cdot \mathrm{s}^{-1}$. Emulator work was gradually increased over several additional walking bouts to acclimate participants to active ankle push-off. The prosthesis was then realigned if necessary. On the second and third acclimation days, participants experienced the full range of emulator work conditions for three minutes per condition. This allowed participants to become comfortable walking on the treadmill in the laboratory with the prosthesis emulator.

\subsubsection{Training period}

After acclimation, participants completed two training sessions on separate days. Training consisted of Quiet Standing for 3.5 minutes; Prescribed walking for 5 minutes; and each emulator push-off work condition, presented in random order, while walking for 6 minutes each. Participants and experimenters interacting with participants were blinded to emulator conditions. Participants were given 5 minutes of rest between trials. Training sessions allowed participants to adapt to each push-off work condition.

\subsubsection{Collection period}

After training, subjects completed two collection sessions on different days. The protocol was identical to the training period, except that indirect calorimetery, motion capture, force plate, electromyography, and survey data were collected. The first collection day allowed participants to become comfortable with this equipment. Data presented are from the final collection day.

\subsection{Outcome measures}

\subsubsection{Prosthesis mechanics}

Prosthetic ankle angle was measured with an encoder, and was differentiated and low-pass filtered with a cutoff frequency of $60 \mathrm{~Hz}$ to obtain ankle velocity. Prosthetic ankle torque was calculated based on deflection of a series spring (Caputo and Collins, 2014a). Prosthetic ankle power was calculated as the product of velocity and torque. Net prosthesis work was calculated as the integral of ankle power. We present these values as work divided by stride time to facilitate comparison with metabolic rate, and normalized to body mass to facilitate comparison across subjects. Results are presented with units of $\mathrm{J} \cdot \mathrm{kg}^{-1} \cdot \mathrm{s}^{-1}$, rather than $\mathrm{W} \cdot \mathrm{kg}^{-1}$, to avoid confusion with instantaneous ankle joint power.

\subsubsection{Metabolic rate}


Metabolic rate was measured using indirect calorimetry. Subjects fasted for four hours prior to data collection. Rates of oxygen uptake and carbon dioxide production were measured during the final three minutes of each trial by a commercial respirometry system (Oxycon Mobile, CareFusion), and then averaged. Energy consumption was calculated using a standard equation (Brockway, 1987). Change in metabolic rate was calculated by subtracting the value for Prescribed. Values were normalized to body mass to facilitate comparison across subjects. Data were not obtained for Subject 3 in the Highest condition due to a hardware malfunction.

\subsubsection{Center of mass mechanics}

Ground reaction forces were measured using a commercial instrumented split-belt treadmill (Bertec). We used the individual limbs method (Donelan et al., 2002) to calculate center-of-mass power for each leg. We defined phases of center-of-mass power based on gait events and percent stride, rather than periods of continuous positive or negative power, because subjects exhibited irregular patterns. We defined center-of-mass 'push-off' as net trailing leg work during double support, 'collision' as net leading leg work during double support, and 'rebound' as net stance leg work during the first portion of single support. We calculated the biological contribution to positive center of mass work as total positive center of mass work over the entire stride minus positive prosthesis work.

\subsubsection{Joint mechanics}

Joint kinematics were recorded by a commercial motion capture system with seven cameras (MX40, Vicon). Reflective markers were placed at 17 bony landmarks on the pelvis, lower limbs and prosthesis (Supplementary Materials). Inverse dynamics analysis (Winter, 1990) was used to calculate joint moments and powers. Derivatives of marker data were low-pass filtered with a cutoff frequency of $7.5 \mathrm{~Hz}$. Segment mass properties were estimated using anthropometry (Drillis et al, 1964; de Lava, 1996) and emulator mass properties were determined empirically.

\subsubsection{Muscle activity}

Muscle activity was measured using a commercial surface electromyography system (Trigno Wireless, Delsys) from biceps femoris, rectus femoris, and vastus medialis for both legs and gastrocnemius medialis and lateralis, soleus, and tibialis anterior for the intact leg. Data were high-pass filtered with a cutoff frequency of $20 \mathrm{~Hz}$, rectified, and low-pass filtered with a cutoff frequency of $6 \mathrm{~Hz}$ in post processing (Ferris et al., 2006). Erroneous signals due to sensor malfunction, accounting for $8 \%$ of all electromyographic data, were discarded. Muscle activity was normalized to the maximum value recorded during Prescribed walking for each muscle. Muscle activity during periods of interest was integrated and compared across conditions.

\subsubsection{User satisfaction}


Participants were asked to rate each push-off work condition on a scale of 1 to 5 , with 1 being worst and 5 being best, directly following that condition.

\subsection{Statistical analysis}

Statistical comparisons were made of metabolic rate, joint mechanics, center-of-mass mechanics, muscle activity, and user satisfaction. Mechanics and muscle activity trajectories were divided into strides, normalized by stride length, and averaged across strides. Scalar values, such as work, were calculated for individual strides, then averaged. Data were then averaged across participants, and study means and inter-subject variability reported.

We tested for a main effect of net prosthesis work rate on each outcome using repeated measures ANOVA with a significance level of $p \leq 0.05$. All $p$-values refer to ANOVA. Regression was used to characterize the relationship between mean values of net prosthesis work rate, metabolic rate and user satisfaction. Reflecting the three hypothesized relationships, we fit quadratic, linear and exponential models and report results for the model that explained the most variance (Tukey et al., 1985).

\section{Results}

\subsection{Prosthesis mechanics}

Positive ankle push-off work rate ranged from 0.08 to $0.39 \mathrm{~J} \cdot \mathrm{kg}^{-1} \cdot \mathrm{s}^{-1}$, or from about half to about double the value from non-amputee walking. Net prosthesis work rate ranged from -0.06 to 0.26 $\mathrm{J} \cdot \mathrm{kg}^{-1} \cdot \mathrm{s}^{-1}$, or about -2 to 10 times the value from non-amputee walking. One participant (Subject 6) adapted to the emulator in a way that prevented large amounts of work production, but still experienced a range of net prosthesis work. Prosthetic ankle power (Fig. 2) and torque-angle curves (Fig. S3) had patterns similar to the intact ankle and other active prostheses. Push-off onset and peak timing were consistent within $3 \%$ across conditions. Negative work rate was unchanged $(p=0.7)$, while stride period was always within $0.02 \mathrm{~s}$ of the value for Prescribed $(1.13 \mathrm{~s})$. Thus, changes in positive push-off work were equivalent to changes in net prosthesis work rate.

\subsection{Metabolic rate}

Net prosthesis work rate did not significantly affect metabolic rate (Fig. $3 ; p=0.5$ ). Regression showed no relationship (quadratic fit: $\mathrm{R}^{2}=0.007$; exponential fit: $\mathrm{R}^{2}=0.002$; linear fit: $\mathrm{R}^{2}=$ 0.0003). Effects were inconsistent across participants, but no participant tended towards lower metabolic rate with increasing prosthetic ankle push-off work (Fig. 4). Only one participant consistently exhibited lower metabolic rate than with their Prescribed foot, achieved with about zero net prosthesis work (Fig. 4, Subject 6). Metabolic rate in Prescribed was $2.8 \pm 0.6 \mathrm{~J} \cdot \mathrm{kg}^{-1} \cdot \mathrm{s}^{-1}$.

\subsection{Gait mechanics and muscle activity}


Center-of-mass push-off work rate was strongly affected by net prosthesis work rate (Fig. 5; $\left.p=8 \cdot 10^{-8}\right)$, but intact-limb collision work rate was unaffected $(p=0.9)$. Intact-limb rebound work rate decreased $\left(p=2 \cdot 10^{-3}\right)$ and total positive center-of-mass work rate attributed to the human decreased substantially $\left(p=3 \cdot 10^{-3}\right)$ with increasing push-off. Consistent trends were observed in ground reaction forces (Fig. S5).

Positive prosthesis-side hip power during swing initiation decreased substantially with increasing prosthesis work (Fig. 6; $p=0.06$ ). The pattern of prosthesis-side knee power during swing seems to have been altered (Fig. S4), but negative knee work during swing was unchanged ( $p=1.0)$.

Biceps femoris activity in the prosthesis-side limb during push-off increased substantially with increasing push-off work (Fig. 7; $p=0.07$ ). Biceps femoris activity in the intact limb during early intact stance decreased slightly with increasing push-off work (Fig. $8 ; p=7 \cdot 10^{-3}$ ).

Center-of-mass power (Fig. 5), joint mechanics (Figs. S6-8), and muscle activity (Fig. S9-10) showed no other trends, but varied widely across subjects (Supplementary Data; Fig. S11).

\subsection{User satisfaction}

User rating was strongly affected by net prosthesis work rate $\left(p=9 \cdot 10^{-4}\right)$, well characterized by a quadratic model (Fig. 9; $\mathrm{R}^{2}=0.97$ ). Linear and exponential models fit less well $\left(\mathrm{R}^{2}=0.5\right.$, both). Satisfaction was maximized by Medium-Low net prosthesis work, followed closely by Medium.

\subsection{Supplementary data}

Complete results from this experiment are provided in a Supplementary Data file archive.

\section{Discussion}

We hypothesized that prosthetic ankle push-off work would affect the metabolic cost of walking for unilateral transtibial amputees. Our results do not support this hypothesis; a wide range of push-off work was applied, but metabolic rate was not significantly affected. Restoring or augmenting ankle push-off work is therefore not sufficient to improve energy economy for lowerlimb amputees. Other factors such as prosthesis control, push-off timing, training, or subject characteristics must be involved with, or independently responsible for, the apparent benefits of active prostheses.

\subsection{Underlying causes}

Metabolic rate did not covary with push-off work despite underlying trends that would suggest beneficial changes in gait mechanics. As net prosthesis work rate increased, we measured reductions in the human contribution to total positive center of mass work, hip work associated with prosthesis-side swing initiation, and biceps femoris activity in the intact limb during prosthesis push-off. Additional costs might have been incurred in the prosthesis-side knee during swing initiation, indicated by increased biceps femoris activity, but both overall knee work and 
knee flexion moment were unchanged. The complex relationships between prosthesis behavior, gait mechanics, muscle activity and metabolic energy consumption remain an important area of research.

Net prosthesis work rate does not define all prosthesis mechanics, and the effects of push-off work on metabolic rate might differ with alternate baseline mechanics. Ankle power and torqueangle curves in this study were similar to those in prior studies of active prostheses, but with some differences. Consistent with prior active devices (Esposito et al., 2015), inverse-dynamics analysis showed less negative prosthesis work and earlier onset of positive work than with prescribed feet (Fig. 2). However, onboard measurements of power (Fig. S2) and torque-angle curves (Fig. S3) depicted a smoother increase in power during push-off (Au, 2007) and a more circular work loop (Herr \& Grabowski, 2012) than prior active devices. Interactions between these features and net prosthesis work should be explored in future studies.

\subsection{Study limitations}

This study was designed to observe population-wide trends in metabolic rate with push-off work, but individual responses did not suggest a consistent trend. A study design that instead optimized prosthesis function for each user and then measured net prosthesis work in optimized conditions would provide additional insights. Such a process might be responsible for previously-measured benefits with active prostheses whose control properties were hand-tuned for each user during fitting (Herr and Grabowski, 2012). Nonetheless, mean data in the present study do not show a trend, supporting the conclusion that increasing ankle push-off work with a powered prosthesis does not necessarily reduce metabolic rate.

Participants seem to have been provided sufficient time to train in each push-off work condition, but alternate training might have altered results. Participants were given six days of acclimation and training before data collection, accumulating more than 30 minutes of walking under each push-off work condition and more than 200 minutes of walking with the prosthesis emulator. In studies with active exoskeletons, 20 minutes of training has been sufficient to reach steady state (Gordon and Ferris, 2007; Galle et al., 2013). To verify that participants had received sufficient training, we conducted additional collections with one subject (Subject 1; Fig. S12) for a total of 10 data collections. At the end of this period, the relationship between push-off work and metabolic rate was unchanged. Nevertheless, it is possible that more training, enforced exploration or coaching could have led participants to use push-off work more effectively. In prior studies with active prostheses, participants had acclimation periods of several weeks (Herr and Grabowski, 2012; Esposito et al., 2015). Future work should address accommodation time and other factors affecting adaptation.

Only six participants were tested due to recruitment challenges. Despite this relatively small sample size, a post-hoc analysis showed statistical power of $70 \%$ to discriminate $20 \%$ changes in 
metabolic rate. A larger sample size would have strengthened our findings, but it is unlikely that our results would be reversed because no participant exhibited the hypothesized trends.

\subsection{Hypotheses revisited}

Although Medium levels of ankle push-off work resulted in a better approximation of this aspect of biological ankle function, doing so did not improve energy cost. Perhaps an imitation of some other aspect of ankle function would provide a benefit. However, altered actuation, sensing, and control as a result of amputation make completely biomimetic function difficult and possibly undesirable. For example, coordination between ankle and knee joints is afforded by biarticular musculature but not ankle prostheses, which can lead to differences in theoretically-optimal control patterns (Handford \& Srinivasan, 2016). As another example, loading the residual limb in a manner similar to non-amputee walking may cause pain, leading to effortful compensations. This is inconsistent with the hypothesis that matching normal ankle work is energetically optimal.

Increasing net prosthesis work reduced positive work performed by the human, but did not reduce metabolic rate. The connection between mechanical work and metabolic rate during steady walking is complex; tendons perform a substantial portion of joint work (Ishikawa et al., 2005), while muscles consume energy for functions other than work production (Umberger et al., 2003). Reducing limb work therefore need not reduce metabolic rate (Jackson and Collins, 2015). This might help explain findings of powered ankle-foot prostheses not reducing metabolic rate during the work-intensive task of walking uphill (Esposito et al., 2015). Complementarily, the energy cost of walking can be reduced with entirely passive devices (Collins et al., 2015). These results conflict with the hypothesis that augmentation arises in proportion to net energy input, offset by the cost of carried mass (Mooney et al., 2014).

Increased prosthesis push-off work did not reduce intact-limb collision work, contrary to predictions based on simple models. Inverted pendulum models require a relationship between push-off and collision impulses, but humans have other means of directing the center of mass during single support. As a result, prior studies have shown that push-off can be inhibited (Vanderpool et al. 2008) or augmented (Kerkum et al., 2015) without affecting metabolic rate. Likewise, the human contribution to center-of-mass work rate decreased with increasing push-off, but this did not reduce metabolic rate. The connections between mechanical work and metabolic energy use are complex. For example, changes in objectives could alter amputee muscle activity and metabolic rate even if joint kinematics and kinetics were to remain fixed (Fey et al. 2012). This conflicts with the hypothesis that reducing center-of-mass work reduces metabolic rate.

Our results do not confirm the relationship between push-off work and metabolic rate that might be inferred from prior comparisons of active and passive prostheses. Restoring push-off work, by itself, is insufficient to normalize metabolic rate. Active push-off might only be effective in the presence of other prosthesis features or control, such as high mass or positive force feedback. Active prostheses might provide benefits only to amputees with particular deficits. For example, 
high-fitness amputees using passive prostheses need not have higher metabolic rate than nonamputees (Esposito et al., 2014) despite having no active push-off. It is even possible that other factors are entirely responsible for previously-observed benefits. Balance provides one possible explanation. Controllers that enhance balance by varying prosthesis push-off work on each step can substantially reduce metabolic rate compared to static controllers with the same average push-off work (Kim and Collins, 2015). Reflex-like controllers used in other active prostheses (Eilenberg et al., 2010) provide enhanced balance in simulation (Song \& Geyer, 2015). Future research should identify which features are responsible for the benefits of active prostheses.

Our results are inconsistent with the relationship between push-off work and metabolic rate observed for non-amputees using a prosthesis simulator. Population differences, particularly in limb mass properties, musculature, proprioception, socket loading and control, and pain, are likely responsible for the qualitative differences in observed trends. On the basis of this and other comparisons (Zelik et al., 2011) it is ill-advised to interpret simulated amputation results as relevant to amputees.

\subsection{Motivations revisited}

Reducing the metabolic cost of amputee gait would improve mobility, but is not the only important objective of prosthesis design. In the present study, push-off work did not affect metabolic rate but strongly affected user satisfaction, indicating alternate benefits that might relate to sense of effort, balance, comfort or appearance. The potential for active prostheses to enhance these outcomes should receive more attention during their design.

\section{Acknowledgements}

The authors thank Mary Ann Miknevich for assistance with recruitment, Hartmut Geyer for use of laboratory equipment, Bambi Brewer for assistance with prosthesis fittings, and Madeleine Brancaccio, Calvin Chan, Nicole Huang, Jenna Krug, Jonathon Lin, Margaret Anne Smith, and Jordan Zile for assistance with data collection. This material is based upon work supported by the National Science Foundation under Grant No. CMMI-1300804.

\section{Conflict of Interest}

The authors have no relationships - financial, personal, or otherwise - that could inappropriately bias the work presented here.

\section{References}

Au, S. K., 2007. Powered Ankle-Foot Prosthesis for the Improvement of Amputee Walking Economy. Doctoral Dissertation, Massachusetts Institute of Technology, cf. Figure 5-13. 
Au, S. K., Weber, J., and Herr, H., 2007. Biomechanical design of a powered ankle-foot prosthesis. In Proceedings of the International Conference on Rehabilitation Robotics (ICORR), pages 298-303.

Brockway, J. M., 1987. Derivation of formulae used to calculate energy expenditure in man. Human Nutrition: Clinical Nutrition 41C, 463-471.

Caputo, J. M., and Collins, S. H., 2014a. A universal ankle-foot prosthesis emulator for experiments during human locomotion. Journal of Biomechanical Engineering 136, 035002.

Caputo, J. M., and Collins, S. H., 2014b. Prosthetic ankle push-off work reduces metabolic rate but not collision work in non-amputee walking. Nature Scientific Reports 4, 7213.

Collins, S. H., Kim, M., Chen, T., and Chen, T., 2015. An ankle-foot prosthesis emulator with control of plantarflexion and inversion-eversion torque. In Proceedings of the International Conference on Robotics and Automation (ICRA), pages 1210-1216.

Collins, S. H., and Kuo, A. D., 2010. Recycling energy to restore impaired ankle function during human walking. Public Library of Science: ONE 5, e9307.

de Lava, P., 1996. Adjustments to Zatsiorsky-Seluyanov's segment inertia parameters. Journal of Biomechanics 29, 1223-1230.

Donelan, J. M., Kram, R., and Kuo, A. D., 2002. Mechanical work for step-to-step transitions is a major determinant of the metabolic cost of human walking. Journal of Experimental Biology 205, 3717-3727.

Drillis, R., Contini, R., and Bluestein, M., 1964. Body segment parameters: A survey of measurement techniques. Artificial Limbs 25, 44-66.

Eilenberg, M. F., Geyer, H., and Herr, H., 2010. Control of a powered ankle-foot prosthesis based on a neuromuscular model. Transactions on Neural Systems and Rehabilitation Engineering 18, 164-173.

Esposito, E. R., Rodriguez, K. M., Ràbago, C. A., and Wilken, J. M., 2014. Does unilateral transtibial amputation lead to greater metabolic demand during walking? Journal of Rehabilitation Research and Development 51, 1287.

Esposito, E. R., Whitehead, J. M. A., and Wiken, J. M., 2015. Step-to-step transition work during level and inclined walking using passive and powered ankle-foot prostheses. Prosthetics and Orthotics International, 0309364614564021.

Ferris, D. P., Gordon, K. E., and Sawicki, G. S., 2006. An improved powered ankle-foot orthosis using proportional myoelectric control. Gait \& Posture 23, 425-428. 
Fey, N. P., Klute, G. K., and Neptune, R. R., 2012. Optimization of prosthetic foot stiffness to reduce metabolic cost and intact knee loading during below-knee amputee walking: a theoretical study. Journal of Biomechanical Engineering 134, 111005.

Fregly, B. J., Besier, T. F., Lloyd, D. G., Delp, S. L., Banks, S. A., Pandy, M. G., and D'Lima, D. D., 2012. Grand challenge competition to predict in vivo knee loads. Journal of Orthopaedic Research 30, 503-513.

Gitter, A., Czerniecki, J. M., and DeGroot, D. M., 1991. Biomechanical analysis of the influence of prosthetic feet on below-knee amputee walking. American Journal of Physical Medicine \& Rehabilitation 70, 142-148.

Goldfarb, M., Lawson, B. E., and Schultz, A. H., 2013. Realizing the promise of robotic leg prostheses. Science Translational Medicine 5, 1-6.

Gordon, K. E., and Ferris, D. P., 2007. Learning to walk with a robotic ankle exoskeleton. Journal of Biomechanics 40, 2636-2644.

Handford, M. L., and Srinivasan, M., 2016. Robotic lower limb prosthesis design through simultaneous computer optimizations of human and prosthesis costs. Scientific Reports 6, 19983.

Herr, H. M., and Grabowski, A. M., 2012. Bionic ankle-foot prosthesis normalizes walking gait for persons with leg amputation. Proceedings of the Royal Society of London B: Biological Sciences 279, 457-464.

Houdijk, H., Pollmann, E., Groenwald, M., Wiggerts, H., and Polomski, W., 2009. The energy cost for the step-to-step transition in amputee walking. Gait \& Posture 30, 35-40.

Ishikawa, M., Komi, P. V., Grey, M. J., Lepola, V., and Bruggemann, G. P., 2005. Muscletendon interaction and elastic energy usage in human walking. Journal of Applied Physiology 99, 603-608.

Jackson, R. W., and Collins, S. H., 2015. An experimental comparison of the relative benefits of work and torque assistance in ankle exoskeletons. Journal of Applied Physiology 119, 541-557.

Kerkum, Y. L., Buizer, A. I., van den Noort, J. C., Becher, J. G., Harlaar, J., and Brehm, M. A., 2015. The effects of varying ankle foot orthosis stiffness on gait in children with spastic cerebral palsy who walk with excessive knee flexion. Public Library of Science: ONE 10, e0142878.

Kim, M., and Collins, S. H., 2015. Once-per-step control of ankle-foot prosthesis push-off work reduces effort associated with balance during walking. Journal of NeuroEngineering and Rehabilitation 12, 43.

Kuo, A. D., Donelan, J. M., and Ruina, A., 2005. Energetic consequences of walking like an inverted pendulum: step-to-step transitions. Exercise and Sport Sciences Reviews 33, 88-97. 
Mooney, L. M., Rouse, E. J., and Herr, H. M., 2014. Autonomous exoskeleton reduces metabolic cost of human walking during load carriage. Journal of NeuroEngineering and Rehabilitation 11, 80.

Segal, A. D., Zelik, K. E., Klute, G. K., Morgenroth, D. C., Hahn, M. E., Orendurff, M. S., Adamczyk, P. G., Collins, S. H., Kuo, A. D., and Czerniecki, J. M., 2012. The effects of a controlled energy storage and return prototype prosthetic foot on transtibial amputee ambulation. Human Movement Science 31, 918-931.

Shultz, A. H., Mitchell, J. E., Truex, D., Lawson, B. E., and Goldfarb, M., 2013. Preliminary evaluation of a walking controller for a powered ankle prosthesis. In Proceedings of the International Conference on Robotics and Automation, pages 4838-4843.

Song, S., and Geyer, H., 2015. A neural circuitry that emphasizes spinal feedback generates diverse behaviours of human locomotion. The Journal of Physiology 593, 3493-3511.

Tukey, J.W., Ciminera, J. L., and Heyse, J. F., 1984. Testing the statistical certainty of a response to increasing doses of a drug. Biometrics 41, 295-301.

Umberger, B. R., Gerritsen, K. G. M., and Martin, P. E., 2003. A model of human muscle energy expenditure. Computer Methods in Biomechanics and Biomedical Engineering 6, 99-111.

Vanderpool, M. T., Collins, S. H., and Kuo, A. D., 2008. Ankle fixation need not increase the energetic cost of human walking. Gait \& Posture 28, 427-433.

Waters, R. L., and Mulroy, S., 1999. The energy expenditure of normal and pathologic gait. Gait \& Posture 9, 207-231.

Winter, D. A., 1990. Biomechanics and Motor Control of Human Movement. John Wiley \& Sons, Inc., Toronto.

Winter, D. A., 1991. The Biomechanics and Motor Control of Human Gait: Normal, Elderly and Pathological. Waterloo Biomechanics, Waterloo.

Zelik, K. E., Collins, S. H., Adamczyk, P. G., Segal, A. D., Klute, G. K., Morgenroth, D. C., Hahn, M. E., Orendurff, M. S., Czerniecki, J. M., and Kuo, A. D., 2011. Systematic variation of prosthetic foot parameter affects center-of-mass mechanics and metabolic cost during walking. Transactions on Neural Systems and Rehabilitation Engineering 19, 411-419.

Zhang, J., Cheah, C. C., and Collins, S. H., 2015. Experimental comparison of torque control methods on an ankle exoskeleton during human walking. In Proceedings of the International Conference on Robotics and Automation, pages 5584-5589. 
Zidarov, D., Swaine, B., and Gauthier-Gagnon, C., 2009. Quality of life of persons with lowerlimb amputation during rehabilitation and at 3-month follow-up. Archives of Physical Medicine and Rehabilitation 90, 634-645.

Ziegler-Graham, K., MacKenzie, E. J., Ephraim, P. L., Travison, T. G., and Brookmeyer, R., 2008. Estimating the prevalence of limb loss in the United States: 2005 to 2050. Archives of Physical Medicine and Rehabilitation 89, 422-429. 
Fig. 1. Subjects with unilateral transtibial amputation wore the tethered ankle-foot prosthesis emulator while walking on a treadmill. A description of the emulator and photograph of the prosthesis are provided as supplementary materials (Fig. S1). Ankle push-off work was varied while metabolic rate, gait mechanics, muscle activity and user satisfaction were recorded.

Fig. 2. Peak prosthetic ankle power during push-off ranged from about half to about double the value for subjects' Prescribed feet. Negative power, push-off onset, and the timing of peak power were consistent across emulator conditions. The onset of positive power was earlier than with Prescribed feet, consistent with other active prostheses (Esposito et al., 2015). Power shown here was estimated using inverse-dynamics analysis to facilitate comparisons. Values from onboard sensors are provided in Fig. S2.

Fig. 3. Metabolic rate was not affected by prosthesis push-off work. Net prosthesis work rate spanned a wide range, including values approximating those of subjects' prescribed feet in this study (Prescribed, dashed line at left), the normal biological ankle (dashed line at center; from Caputo \& Collins, 2014b) and the BiOM active ankle-foot prostheses (dashed line at right; from Herr \& Grabowski, 2012). Change in metabolic rate compared to Prescribed was not affected by net prosthesis work rate. Regression to mean data using a quadratic model (solid line) accounted for almost none of the variance in metabolic rate. Dots are means. Whiskers denote standard error in metabolic rate (vertical) and net prosthesis work rate (horizontal).

Fig. 4. The effects of prosthesis push-off work on metabolic rate varied across subjects, but no subjects exhibited lower metabolic rate with higher levels of push-off. A quadratic model was fit to data from each participant (solid lines) with corresponding $\mathrm{R}^{2}$ values provided. Only Subject 6 had consistently lower metabolic rate in active push-off conditions than with their prescribed foot, but metabolic rate was minimized for slightly negative net prosthesis work.

Fig. 5. Center-of-mass power (top) for prosthesis-side (blue) and intact-side (red) limbs. Prosthesis-side center-of-mass push-off work rate was strongly affected by net prosthesis work rate, but intact-side collision work rate was not. Intact-side rebound work rate was reduced with increasing net prosthesis work rate, as was total positive center-of-mass work attributed to the human over the entire stride. Rebound was defined to begin at single support and to end at $82 \%$ stride, based on typical periods of positive power. Bars are mean integrals over the indicated regions, error bars are standard deviations. Results of statistical tests (ANOVA) for an effect of net prosthesis work rate on net work during each phase are shown above corresponding bars.

Fig. 6. Positive power at the prosthesis-side hip during early swing decreased with increasing net prosthesis work. This suggests a reduction in effort associated with swing initiation. Bars are mean integrals over the indicated region, error bars are standard deviations.

Fig. 7. Prosthesis-side biceps femoris activity increased during swing initiation and early swing as net prosthesis work increased. This might indicate resistance to excessive leg swing, although such 
compensation seems to be at odds with joint mechanics data at the hip (Fig. 6) and knee (Fig. S4). Bars are mean integrals over the indicated region, error bars are standard deviations.

Fig. 8. Intact-limb biceps femoris muscle activity decreased during early stance as net prosthesis work increased. This might indicate a reduced reliance on a hip powering strategy during the period of prosthetic ankle push-off. Bars are mean integrals over the indicated region, error bars are standard deviations. $0 \%$ stride refers to prosthesis-side heel strike.

Fig. 9. User satisfaction was strongly affected by net prosthesis work, with Medium-Low being preferred and Medium second-most preferred. Regression to mean data using a quadratic model (solid line) explained most of the variance in preference. Users rated each condition on a scale of 1 to 5 , with 1 being worst and 5 being best. Dots are means. Whiskers denote standard error in metabolic rate (vertical) and net prosthesis work rate (horizontal). 


\section{Figures}

Figure 1

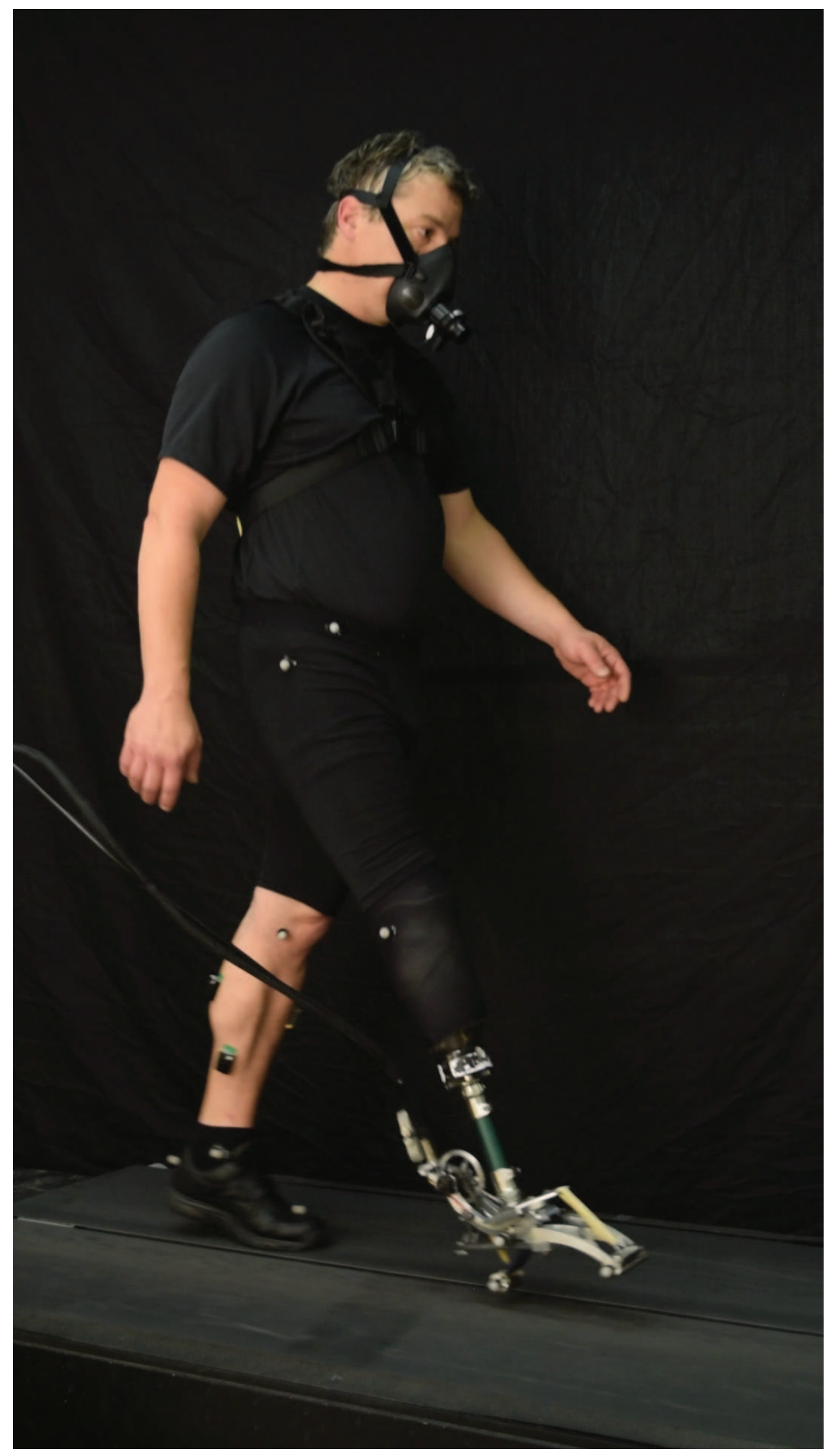


Figure 2

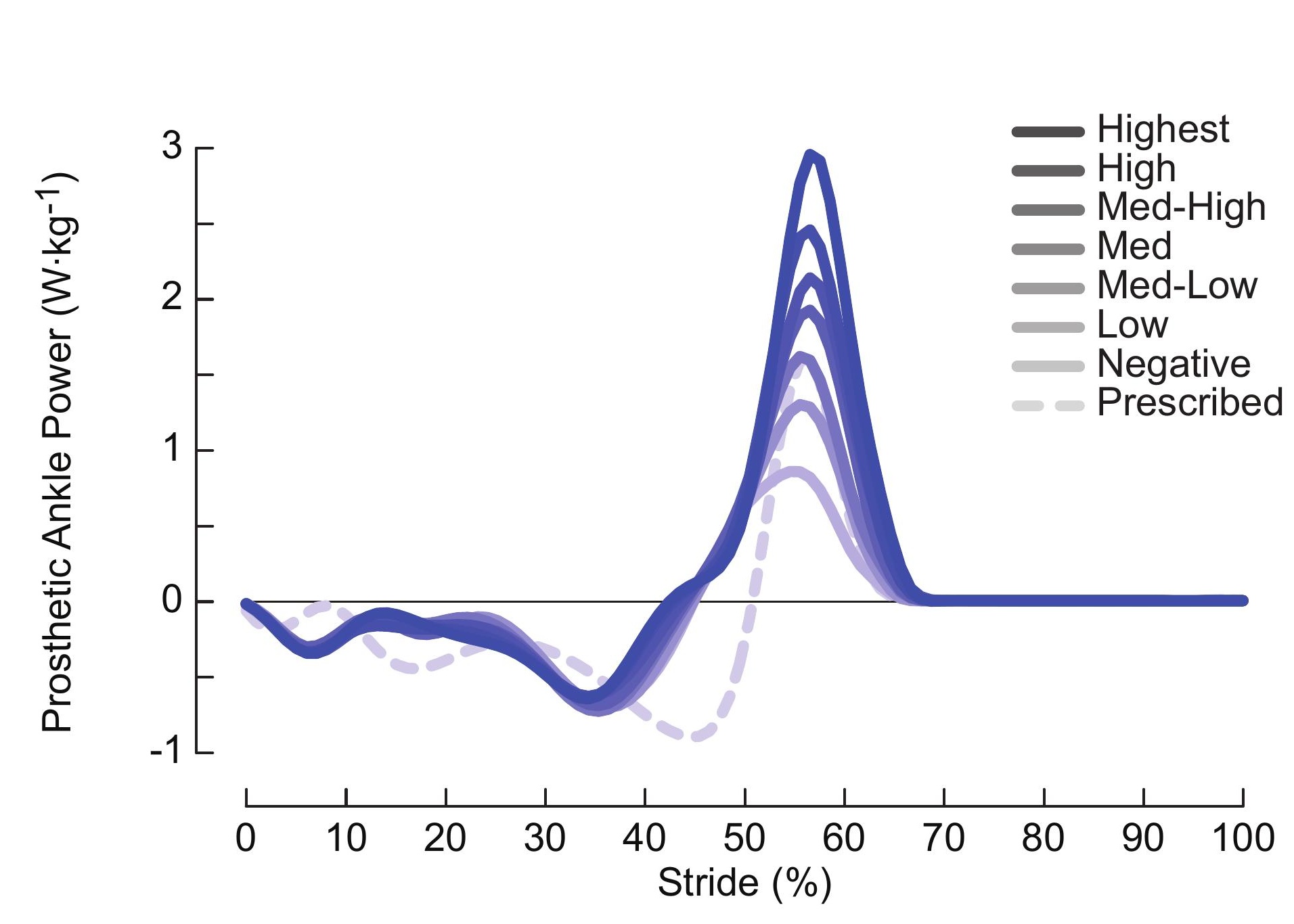

Figure 2 


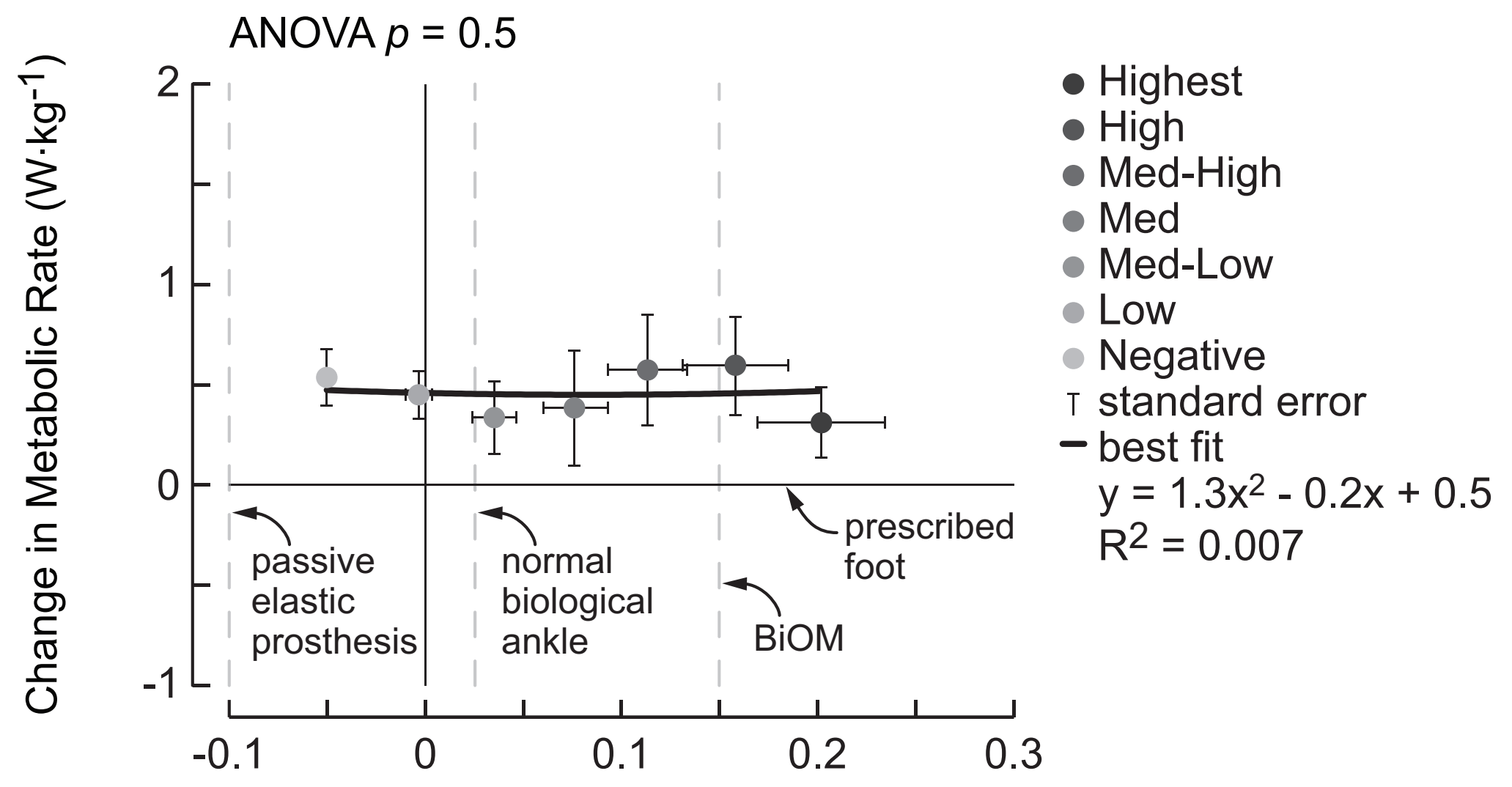

Net prosthesis Work Divided by Stride Period $\left(\mathrm{J} \cdot \mathrm{kg}^{-1} \cdot \mathrm{s}^{-1}\right)$ 
Figure 4

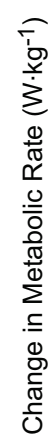
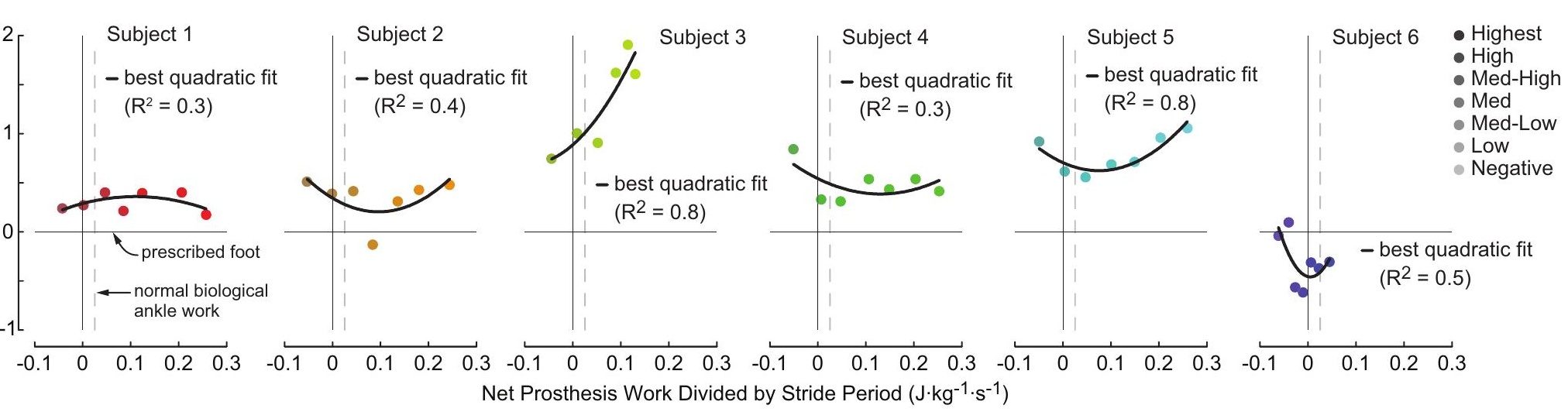
Figure 5
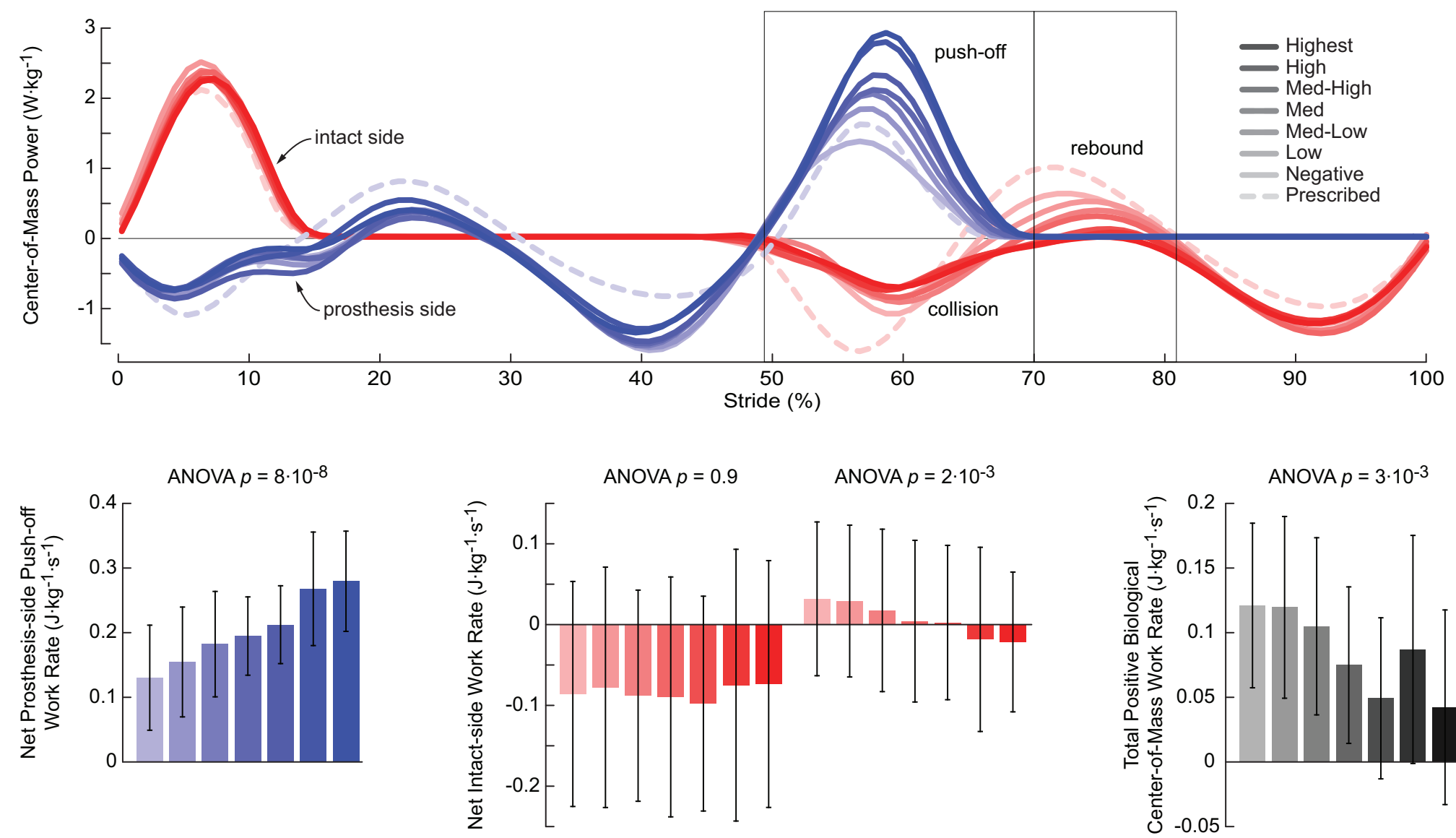

push-off

collision

rebound

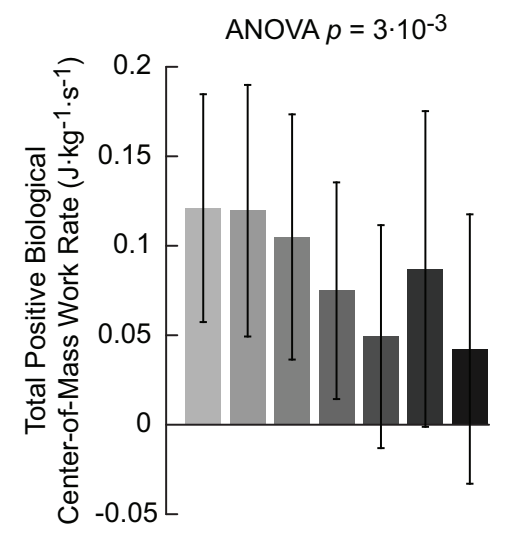

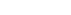



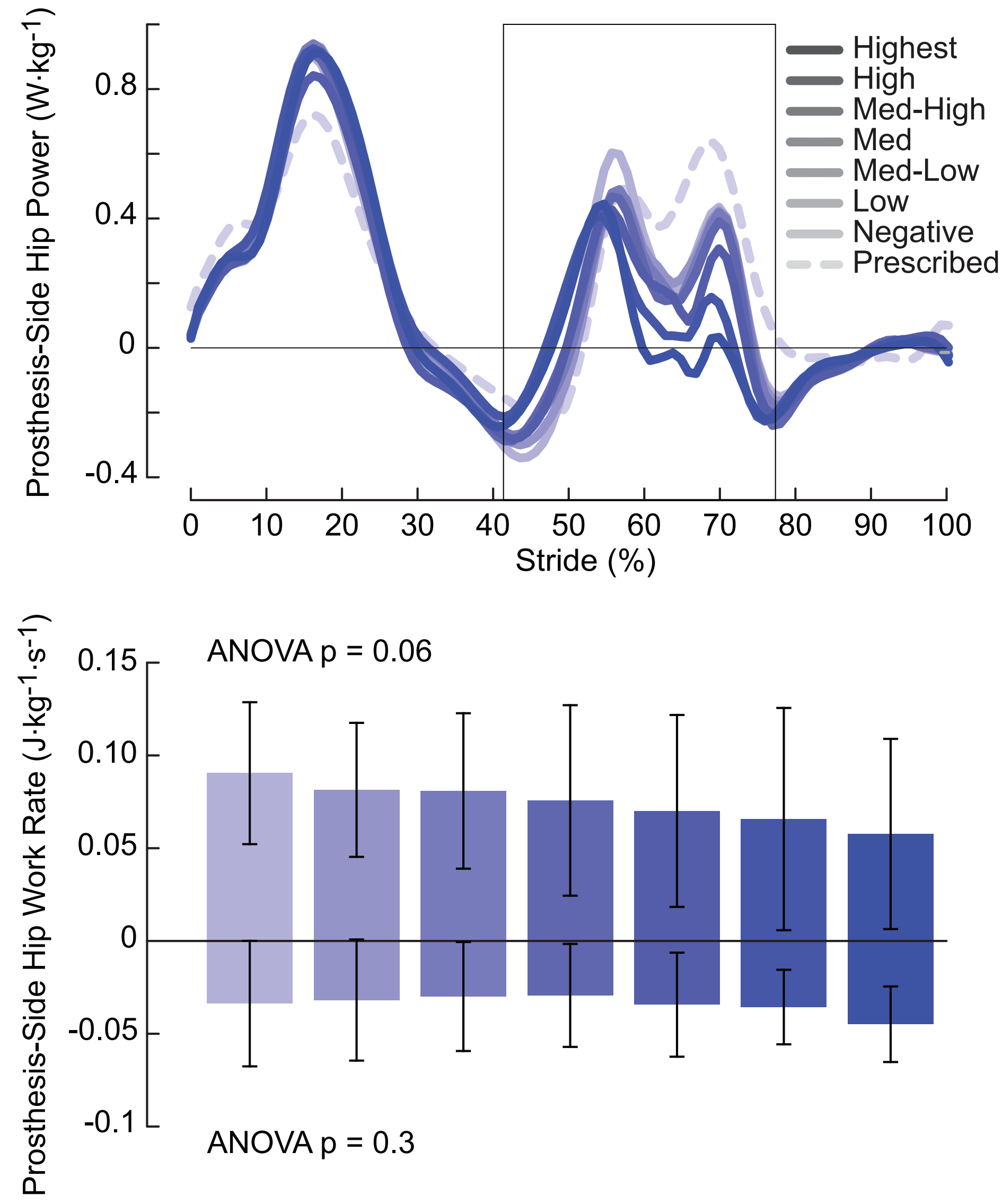

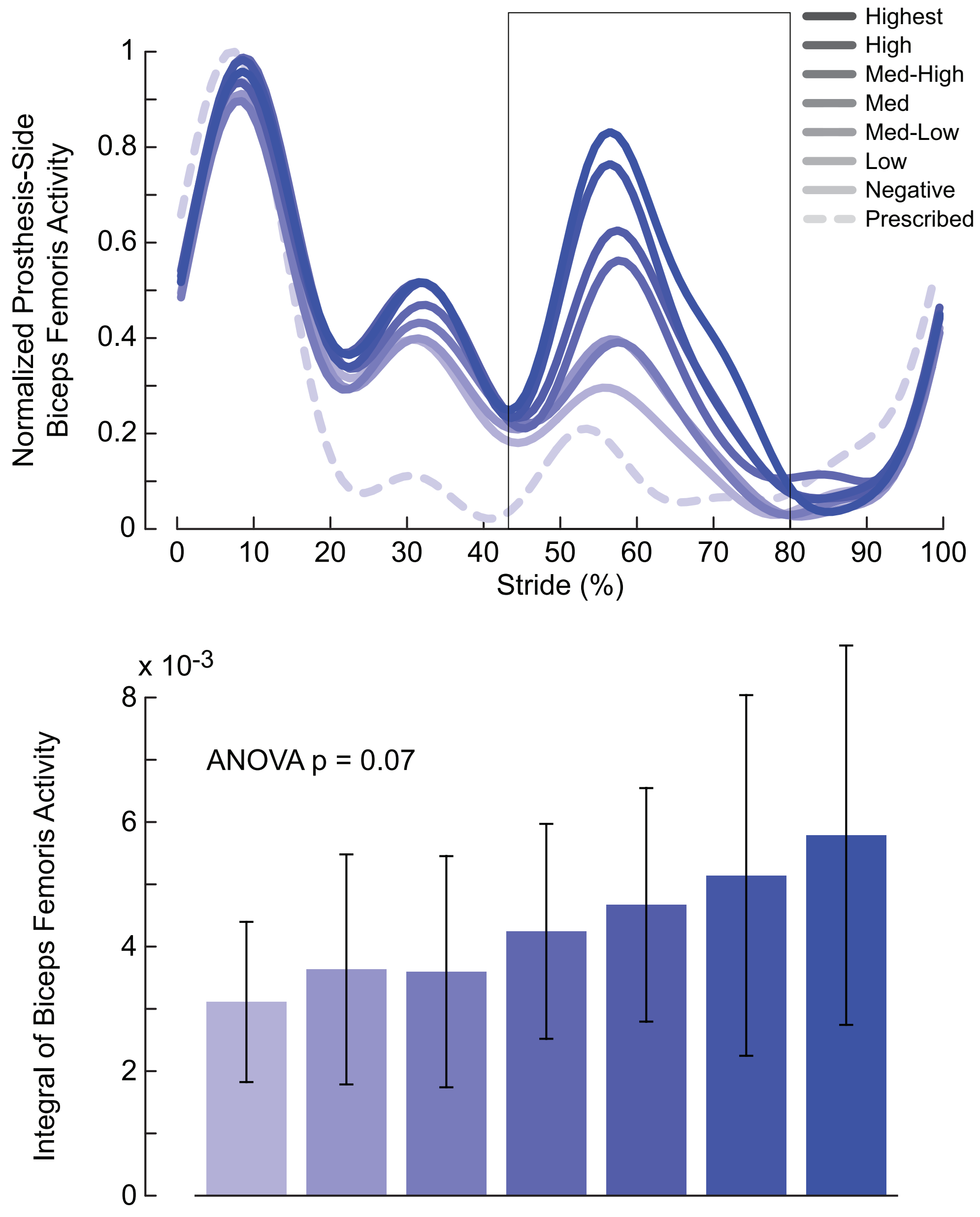

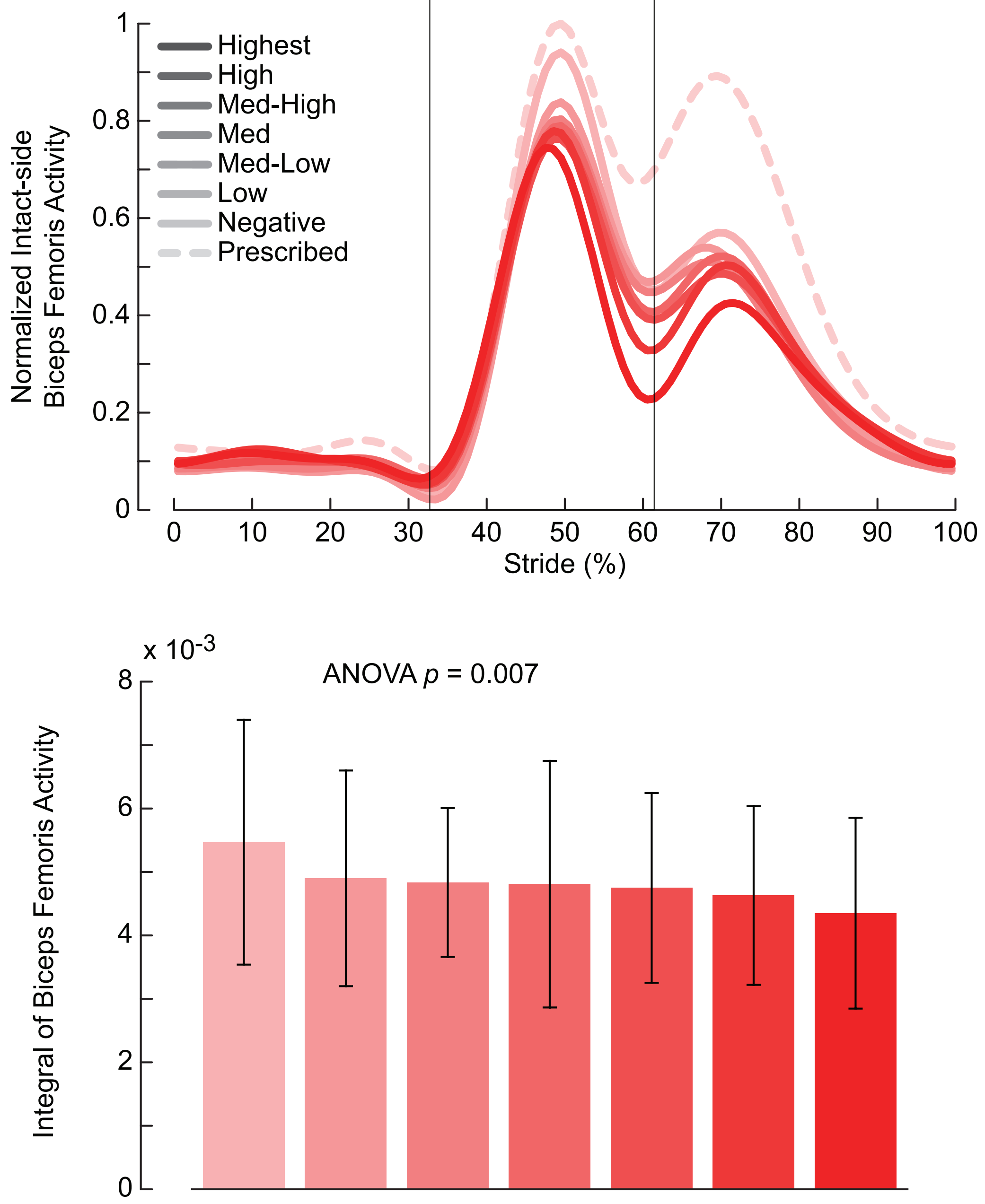


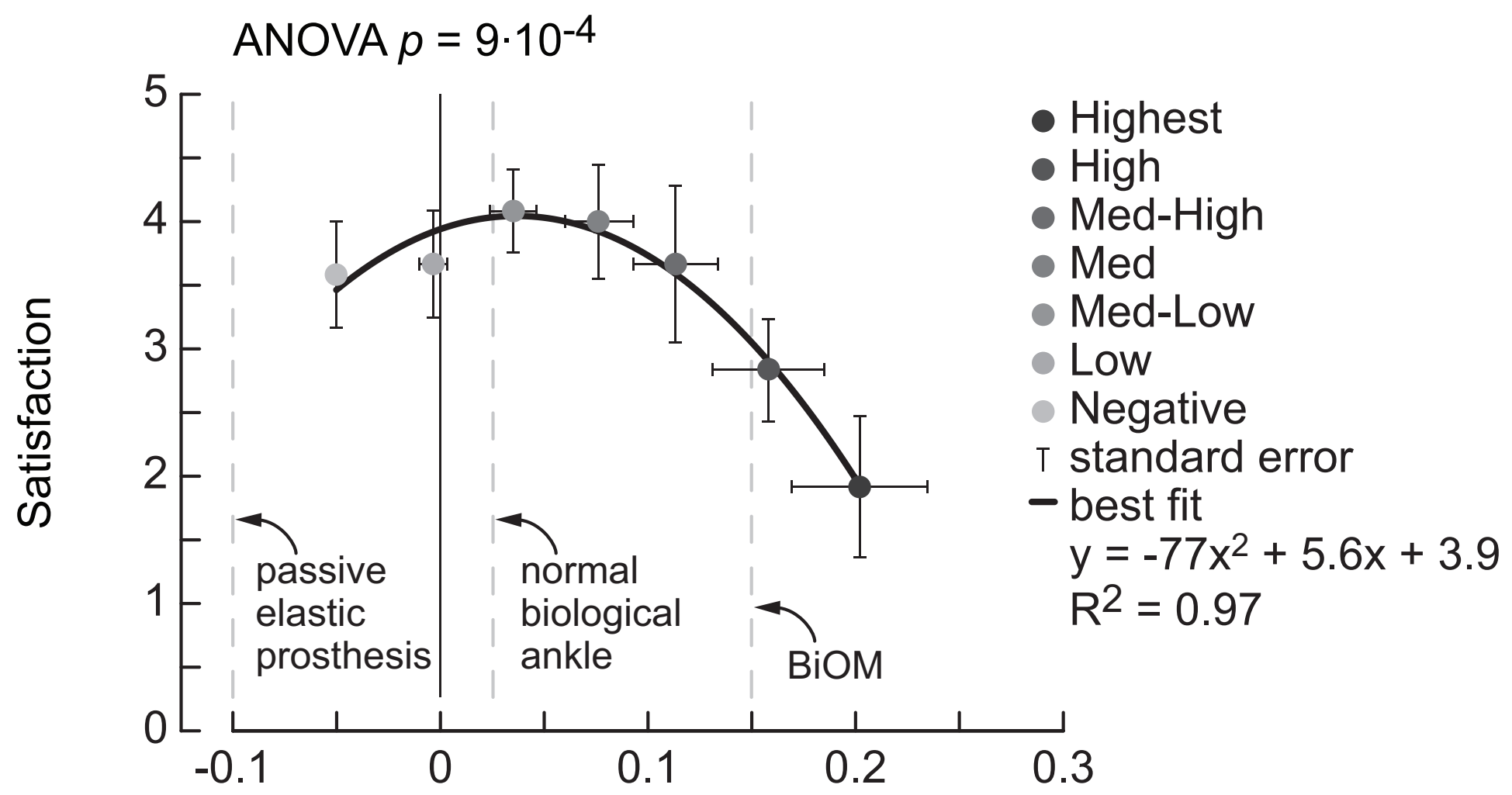

Net Prosthesis Work Divided by Stride Period $\left(\mathrm{J} \cdot \mathrm{kg}^{-1} \cdot \mathrm{s}^{-1}\right)$ 


\begin{tabular}{|c|c|c|c|c|c|c|c|c|}
\hline & Age & Height & Mass & Amputee & Cause & K level & Side & Prescribed prosthesis \\
\hline Participant 1 & $57 \mathrm{yrs}$ & $1.78 \mathrm{~m}$ & $86.1 \mathrm{~kg}$ & 7 yrs & trauma & K3 & left & Ottobock 1C61 Triton Vertical Shock \\
\hline Participant 2 & $45 \mathrm{yrs}$ & $1.78 \mathrm{~m}$ & $86.0 \mathrm{~kg}$ & $1 \mathrm{yrs}$ & trauma & $\mathrm{K} 3$ & right & Össur Vari-Flex \\
\hline Participant 3 & $48 \mathrm{yrs}$ & $1.82 \mathrm{~m}$ & $95.1 \mathrm{~kg}$ & $11 \mathrm{yrs}$ & trauma & K3 & left & $\mathrm{BiOM} \mathrm{T} 2$ \\
\hline Participant 4 & 39 yrs & $1.83 \mathrm{~m}$ & $98.7 \mathrm{~kg}$ & 8 yrs & trauma & K3 & right & Fillauer Wave Sport \\
\hline Participant 5 & 42 yrs & $1.78 \mathrm{~m}$ & $86.9 \mathrm{~kg}$ & 8 yrs & trauma & K3 & right & Fillauer Wave Sport \\
\hline Participant 6 & 49 yrs & $1.73 \mathrm{~m}$ & $74.2 \mathrm{~kg}$ & 45 yrs & congenital & K3 & right & Freedom Innovations Renegade $\mathrm{A} \cdot \mathrm{T}$ \\
\hline
\end{tabular}

